\title{
Group Theory
}

\section{Nilpotent subalgebras of semisimple Lie algebras}

\author{
Paul Levy ${ }^{\mathrm{a}}$, George McNinch ${ }^{\mathrm{b}}$, Donna M. Testerman ${ }^{\mathrm{a}, 1}$ \\ a École polytechnique fédérale de Lausanne, IGAT, bâtiment BCH, CH-1015 Lausanne, Switzerland \\ ${ }^{\mathrm{b}}$ Department of Mathematics, Tufts University, 503, Boston Avenue, Medford, MA 01255, USA
}

Received 2 December 2008; accepted after revision 17 March 2009

Available online 8 April 2009

Presented by Jean-Pierre Serre

\begin{abstract}
Let $\mathfrak{g}$ be the Lie algebra of a semisimple linear algebraic group. Under mild conditions on the characteristic of the underlying field, one can show that any subalgebra of $\mathfrak{g}$ consisting of nilpotent elements is contained in some Borel subalgebra. In this Note, we provide examples for each semisimple group $G$ and for each of the torsion primes for $G$ of nil subalgebras not lying in any Borel subalgebra of $\mathfrak{g}$. To cite this article: P. Levy et al., C. R. Acad. Sci. Paris, Ser. I 347 (2009).

(c) 2009 Published by Elsevier Masson SAS on behalf of Académie des sciences.
\end{abstract}

\section{Résumé}

Sous-algèbres nilpotentes d'algèbres de Lie semi-simples. Soit g l'algèbre de Lie d'un groupe algébrique linéaire semi-simple. Si l'on impose certaines conditions à la caractéristique du corps de définition, on peut montrer que toute sous-algèbre de $\mathfrak{g}$ ne contenant que des éléments nilpotents est contenue dans une sous-algèbre de Borel. Dans cette Note, nous donnons des exemples, pour chaque groupe semi-simple $G$ et pour chaque nombre premier de torsion pour $G$, de sous-algèbres d'éléments nilpotents qui ne sont contenues dans aucune sous-algèbre de Borel de $\mathfrak{g}$. Pour citer cet article : P. Levy et al., C. R. Acad. Sci. Paris, Ser. I 347 (2009).

(ㄷ) 2009 Published by Elsevier Masson SAS on behalf of Académie des sciences.

\section{Version française abrégée}

Soit $k$ un corps algébriquement clos de caractéristique $p>0$. Par «groupe algébrique sur $k$ » nous entendons un schéma en groupes affine de type fini sur $k$. Soit $G$ un groupe algébrique semi-simple défini sur $k$ ( $G$ est lisse et connexe) et soit $U$ un sous-groupe (algébrique) unipotent de $G$. Si $U$ est réduit, on sait que $U$ est contenu dans un sous-groupe de Borel de $G$ (cf. [4, 30.4]). Nous nous intéressons au cas où $U$ n'est pas réduit, plus précisément au cas des $p$-sous-algèbres de Lie de $\operatorname{Lie}(G)$.

Théorème 0.1. Supposons que $p$ ne soit pas un nombre premier de torsion de G. Alors tout sous-groupe unipotent (non nécessairement réduit) de G est contenu dans un sous-groupe de Borel de G.

E-mail addresses: paul.levy@epfl.ch (P. Levy), george.mcninch@tufts.edu (G. McNinch), donna.testerman@epfl.ch (D.M. Testerman).

1 Research supported in part by the Swiss National Science Foundation grant number PP002-68710. 
La démonstration repose essentiellement sur [7, Theorem A].

Théorème 0.2. Supposons que p soit un nombre premier de torsion pour G. Il existe un sous-groupe unipotent de G, de dimension 0 , qui n'est contenu dans aucun sous-groupe de Borel de G.

On démontre ce théorème en construisant des $p$-sous-algèbres de Lie de $\operatorname{Lie}(G)$, formées d'éléments nilpotents, et qui ne sont contenues dans aucune sous-algèbre de Borel. Il y a deux types de constructions :

a) Si $\tilde{G} \rightarrow G$ est le revêtement universel de $G$ et si $p$ divise l'ordre du noyau (schématique) de $\tilde{G} \rightarrow G$, on peut construire une $p$-sous-algèbre commutative de $\operatorname{Lie}(G)$, formée d'éléments nilpotents, dont l'image réciproque dans Lie $(\tilde{G})$ n'est pas commutative; une telle sous-algèbre n'est pas contenue dans une sous-algèbre de Borel de $G$. Lorsque $G$ est simple, l'algèbre ainsi construite est de dimension 2, et elle est annulée par la puissance $p$-ième.

b) Si $p$ est de torsion pour le système de racines de $G$ (par exemple $p=2,3$, ou 5 si $G$ est de type $E_{8}$ ), il existe une $p$-sous-algèbre commutative de $\operatorname{Lie}(G)$, de dimension 3 , annulée par la puissance $p$-ième, et non contenue dans une sous-algèbre de Borel.

\section{Introduction}

Let $k$ be an algebraically closed field of characteristic $p>0$ and let $G$ be a semisimple linear algebraic group over $k$. Let $\mathfrak{g}$ be the Lie algebra of $G$. Under mild conditions on $G$ and $p$ it is straightforward to show that any nil subalgebra of $\mathfrak{g}$, that is, a subalgebra consisting of nilpotent elements, is contained in a Borel subalgebra (see Section 2 below). J.-P. Serre has asked the following question: is it true that if $p$ is a torsion prime for $G$ then there exists a nil subalgebra of $\mathfrak{g}$ which is contained in no Borel subalgebra? In this Note, we establish a positive answer to this question. Moreover, if $p$ is not a torsion prime for $G$, every nil subalgebra of $\mathfrak{g}$ lies in a Borel subalgebra. Our argument in fact applies to the more general setting of unipotent subgroup schemes of a semisimple group scheme over $k$.

We outline two separate cases. First, assume that $G$ is simply connected. The scheme-theoretic center $Z$ of $G$ is a finite group scheme. Now by a Heisenberg-type subalgebra of $\mathfrak{g}$, we mean a $p$-subalgebra which is a central extension of an abelian nil algebra by a 1-dimensional algebra. If $p$ divides the order of $Z$, we exhibit a Heisenbergtype restricted subalgebra of $\mathfrak{g}$ whose center is central in $\mathfrak{g}$. This gives a construction of a suitable nil algebra in $\operatorname{Lie}\left(G_{a d}\right)$, where $G_{a d}$ is the corresponding adjoint group. Secondly, assume $p$ is a torsion prime for the root system of $G$. Then we will exhibit a commutative 3-dimensional restricted nil subalgebra of $\mathfrak{g}$ which is not contained in any Borel subalgebra.

In [3], Draisma, Kraft and Kuttler study subspaces of $\mathfrak{g}$, rather than subalgebras, consisting of nilpotent elements; they exhibit examples in Lie algebras defined over fields of certain small characteristics of subspaces of maximal possible dimension which do not lie in a Borel subalgebra. We refer the reader as well to the article of Vasiu [12] in which he studies normal unipotent subgroup schemes of reductive groups.

\section{Good characteristics}

Throughout this Note, $k$ is an algebraically closed field of characteristic $p>0$. By 'linear algebraic group defined over $k$ ' we mean an affine group scheme of finite type over $k$. Let $G$ be a semisimple linear algebraic group over $k$; in particular, $G$ is a smooth group scheme with restricted Lie algebra $\mathfrak{g}$, the $p$-operation being denoted by $X \mapsto X^{p}$. Let $T$ be a fixed maximal torus of $G, W=W(G, T)$ the Weyl group of $G, \Phi=\Phi(G, T)$ the root system, $\Phi^{+}$a positive system in $\Phi, \Delta=\left\{\alpha_{1}, \ldots, \alpha_{\ell}\right\}$ the corresponding basis and $B \subset G$ the associated Borel subgroup containing $T$. For $\alpha \in \Phi$, let $\alpha^{\vee}$ denote the corresponding coroot. If $\Phi$ is an irreducible root system then there is a unique root of maximal height with respect to $\Delta$, noted here by $\beta$. Write $\beta=\sum_{i=1}^{\ell} m_{i} \alpha_{i}$ and $\beta^{\vee}=\sum_{i=1}^{\ell} m_{i}^{\prime} \alpha_{i}^{\vee}$. Recall that $p$ is bad for $\Phi$ if $m_{i}=p$ for some $i, 1 \leqslant i \leqslant \ell$, and $p$ is torsion for $\Phi$ if $m_{i}^{\prime}=p$ for some $i, 1 \leqslant i \leqslant \ell$. (If the Dynkin diagram is simply-laced then $m_{i}=m_{i}^{\prime}$ for all $i$.) We say that $p$ is $\operatorname{good}$ for $\Phi$ if $p$ is not bad for $\Phi$ and that $p$ is very $\operatorname{good}$ for $\Phi$ if $p$ is good for $\Phi$ and $p \nmid(\ell+1)$ when $\Phi$ is of type $A_{\ell}$. Finally, we will say $p$ is good (respectively, very good) for $G$ if $p$ is good (resp. very good) for every irreducible component of $\Phi=\Phi(G, T)$. We will say that $p$ is bad 
for $G$ if $p$ is bad for some irreducible component of $\Phi$ and that $p$ is torsion for $G$ if $p$ is torsion for some irreducible component of $\Phi$ or $p$ divides the order of the fundamental group of $G$. (See [11] for a discussion of torsion primes.)

Before considering the case of non-torsion primes, we introduce one further definition:

Definition 2.1 ([8, Exposé XVII, 1.1]). An algebraic group $U$ over $k$ is said to be unipotent if $U$ admits a composition series whose successive quotients are isomorphic to some subgroup scheme of the algebraic group $\mathbf{G}_{a}$.

Theorem 2.2. Let $G$ be a semisimple group and $p$ a non-torsion prime for $G$. Let $U$ be a unipotent subgroup scheme of $G$. Then $U$ is contained in a Borel subgroup of $G$.

Proof. Consider first the case where $G$ is of type $A_{\ell}$. The result follows from [8, 3.2, Exposé XVII] and induction if $G=\mathrm{SL}_{\ell+1}$. For the other cases, as $p$ does not divide the order of the fundamental group of $G$, we have a separable isogeny $\pi: \mathrm{SL}_{\ell+1} \rightarrow G$ which induces a bijection on the set of Borel subgroups, whence the result follows.

In case $G=\mathrm{Sp}_{2 \ell}$, we argue similarly: a unipotent subgroup of $G$ fixes a non-zero, isotropic vector in the natural representation of $G$ and again by induction lies in a Borel subgroup of $G$. Indeed, this argument works as well for the orthogonal groups when $p \neq 2$.

Consider now the case where $G=G_{2}$ and $p=3$. By the result for $\mathrm{SO}_{7}$, we know that $U$ fixes a nontrivial singular vector in the action of $G$ on its 7-dimensional orthogonal representation. One checks that the stabilizer of such a vector is a parabolic subgroup of $G_{2}$. Indeed this is clear for the group of $k$-points as the long root parabolic lies in the stabilizer and is a maximal subgroup. One checks directly that the stabilizer in $\mathfrak{g}$ of a maximal vector with respect to the fixed Borel subgroup is indeed a parabolic subalgebra with Levi factor a long root $\mathfrak{s l}_{2}$.

Now consider the case where $p$ is a very good prime for $G$. As $G$ is separably isogenous to a simply connected group, we may take $G$ to be simply connected. Then $G$ satisfies the following so-called standard hypotheses for a reductive group $G$ (cf. $[5,5.8])$ :

- $p$ is good for each irreducible component of the root system of $G$,

- the derived subgroup $(G, G)$ is simply connected, and

- there exists a non-degenerate $G$-equivariant symmetric bilinear form $\kappa: \mathfrak{g} \times \mathfrak{g} \rightarrow k$.

We proceed by induction on $\operatorname{dim} G$, the case where $\operatorname{dim} G=3$ and $G=\mathrm{SL}_{2}$ having been handled above. By [8, 3.5], $U$ has a nontrivial center $Z(U)$ and either there exists $X \in \operatorname{Lie}(Z(U))$ with $X^{p}=0$ and so $U \subset C_{G}(X)$ or there exists $u \in Z(U)$ with $u^{p}=1$ and $U \subset C_{G}(u)$. By [10,3.12] there exists a $G$-equivariant bijective morphism between the variety of nilpotent elements and the variety of unipotent elements; so applying Theorem A of [7] we have that $U$ lies in a proper parabolic subgroup $P$ of $G$. Let $L$ be a Levi subgroup of $P$; then $L$ satisfies the standard hypotheses as well. Taking the image of $U$ in $P / R_{u}(P)$, we obtain a unipotent subgroup scheme of $(L, L)$ which is, by induction on the dimension of $G$, contained in a Borel subgroup $B_{L}$ of $L$. We then have that $B_{L} \cdot R_{u}(P)$ is a Borel subgroup of $G$ containing $U$.

It remains to consider the case where the root system of $G$ is not irreducible and $p$ is not a very good prime for $G$. In this case, $G$ is separably isogenous to a direct product of simply connected almost simple groups, and the result follows as in the case of type $A_{\ell}$ above.

\section{Remarks.}

a) Given an arbitrary nil subalgebra $\mathfrak{n}$ of $\mathfrak{g}$, that is not necessarily a restricted subalgebra, one can check via a faithful representation $\mathfrak{g} \rightarrow \mathfrak{g l}(V)$ that the $p$-closure $\overline{\mathfrak{n}}$ of $\mathfrak{n}$ in $\mathfrak{g}$ is again nil. Assume now that $p$ is a non-torsion prime for $G$. Then by the preceding theorem, the infinitesimal unipotent subgroup scheme $\overline{\mathfrak{n}}$ lies in a Borel subalgebra of $G$ and hence $\mathfrak{n}$ does as well.

b) We note that the conclusion of Theorem 2.2 holds for reduced unipotent subgroup schemes even if the characteristic is a torsion prime for $G$. (See [4, 30.4].)

Before presenting our examples, we fix some additional notation. If $G$ is separably isogenous to a simply connected group then we can and will choose a Chevalley basis $\left\{h_{i}, e_{\alpha}, f_{\alpha}: 1 \leqslant i \leqslant \ell, \alpha \in \Phi^{+}\right\}$for $\mathfrak{g}$, satisfying the usual 
relations. If $G$ is not separably isogenous to a simply connected group, then we can choose $\left\{h_{i}, e_{\alpha}, f_{\alpha}: 1 \leqslant i \leqslant \ell\right.$, $\left.\alpha \in \Phi^{+}\right\}$satisfying the usual Chevalley relations; however, the $h_{i}$ will not be linearly independent and a basis of $\mathfrak{g}$ can be obtained by extending $\left\{h_{i}: 1 \leqslant i \leqslant \ell\right\}$ to a basis of $\operatorname{Lie}(T)$. We use the structure constants given in [9] for $\mathfrak{g}$ of type $F_{4}$; for $\mathfrak{g}$ of type $E_{\ell}$, we use those given in [6]. Our labeling of Dynkin diagrams is taken as in [2]. It will sometimes be convenient to represent roots as the $\ell$-tuple of integers giving the coefficients of the simple roots, arranged as in a Dynkin diagram.

\section{Heisenberg-type subalgebras}

Here we take $G$ to be simply connected. For $G=\mathrm{SL}_{m p}$, let $E_{i j}$ denote the elementary $m p \times m p$ matrix with $(r, s)$ entry $\delta_{i r} \delta_{j s}$. Set $X=\sum_{j=0}^{m-1} \sum_{i=1}^{p-1} E_{j p+i, j p+i+1}$ and $Y=\sum_{j=0}^{m-1} \sum_{i=1}^{p-1} i E_{j p+i+1, j p+i}$. Then $X^{p}=0=Y^{p}$, $[X, Y]=I$ and hence the Lie algebra generated by $X$ and $Y$ is nilpotent.

Similar examples exist for other types with a nontrivial center:

- if $p=2$ and $G=\operatorname{Spin}(2 \ell+1, k)$ then let $X=e_{\alpha_{\ell}}$ and $Y=f_{\alpha_{\ell}}$;

- if $p=2$ and $G=\operatorname{Sp}(2 \ell, k)$ then let $X=\sum_{i=1}^{\lceil\ell / 2\rceil} e_{\alpha_{2 i-1}}$ and $Y=\sum_{1}^{\ell} i f_{\alpha_{i}}$;

- if $p=2$ and $G=\operatorname{Spin}(2 \ell, k)$ then let $X=e_{\alpha_{\ell-1}}+e_{\alpha_{\ell}}$ and $Y=f_{\alpha_{\ell-1}}+f_{\alpha_{\ell}}$;

- if $p=3$ and $G$ is of type $E_{6}$ then let $X=e_{\alpha_{1}}+e_{\alpha_{3}}+e_{\alpha_{5}}+e_{\alpha_{6}}$ and $Y=f_{\alpha_{1}}-f_{\alpha_{3}}+f_{\alpha_{5}}-f_{\alpha_{6}}$;

- if $p=2$ and $G$ is of type $E_{7}$ then let $X=e_{\alpha_{2}}+e_{\alpha_{5}}+e_{\alpha_{7}}$ and $Y=f_{\alpha_{2}}+f_{\alpha_{5}}+f_{\alpha_{7}}$.

In each of the above cases $X^{p}=0=Y^{p}$ and $[X, Y]$ is a nontrivial element of $\mathfrak{z}(\mathfrak{g})$, the center of $\mathfrak{g}$; in particular $[X, Y]$ is a nontrivial semisimple element. Hence there does not exist a Borel subalgebra of $\mathfrak{g}$ which contains both $X$ and $Y$.

Now let $G_{a d}$ denote an adjoint type group with root system $\Phi$ and $\pi: G \rightarrow G_{a d}$ the corresponding central isogeny (cf. $\S 22$ of [1]); then $\operatorname{ker}(\mathrm{d} \pi$ ) is central in $\mathfrak{g}$. Applying 22.6 of [1], we see that $\pi$ induces a bijection between Borel subgroups of $G$ and Borel subgroups of $G_{a d}$. Moreover, by [1, 22.4], d $\pi$ is bijective on nilpotent elements in the unipotent radical of a Borel subgroup. We deduce that there is no Borel subalgebra of $\operatorname{Lie}\left(G_{a d}\right)$ which contains both $\mathrm{d} \pi(X)$ and $\mathrm{d} \pi(Y)$. Setting $\mathfrak{h}=k \mathrm{~d} \pi(X)+k \mathrm{~d} \pi(Y)$, we have our desired example.

Suppose now that the root system of $G$ is not irreducible. Set $X=\sum_{i=1}^{\ell} e_{\alpha_{i}} \in \mathfrak{g}$, so $X \in \operatorname{Lie}(B)$. Then there exists a cocharacter $\tau: \mathbf{G}_{m} \rightarrow T$ with $X$ in $\mathfrak{g}(\tau ; 2)$, the 2-weight space with respect to $\tau$ and $\operatorname{Lie}(B)=\bigoplus_{i \geqslant 0} \mathfrak{g}(\tau ; i)$. In particular, $\operatorname{ad}(X): \mathfrak{g}(\tau ; i) \rightarrow \mathfrak{g}(\tau ; i+2)$ for all $i \in \mathbb{Z}$. It is clear that $\operatorname{ad}(X): \mathfrak{g}(\tau ;-2) \rightarrow \mathfrak{g}(\tau ; 0)=\operatorname{Lie}(T)$ is surjective.

Suppose now that $G_{0}$ is isogenous to $G$ and $p$ divides the order of the fundamental group of $G_{0}$. Let $\pi: G \rightarrow G_{0}$ be a central isogeny; our assumption on $p$ implies that there exists $0 \neq W \in \operatorname{ker}(\mathrm{d} \pi)$. Then $W \in \operatorname{Lie}(T)$; hence there exists a unique $Y \in \mathfrak{g}(\tau ;-2)$ for which $[X, Y]=W$. Set $\mathfrak{h} \subset \operatorname{Lie}\left(G_{0}\right)$ to be the restricted subalgebra generated by $\mathrm{d} \pi(X)$ and $\mathrm{d} \pi(Y)$. The proof that $\mathfrak{h}$ does not lie in any Borel subalgebra of $\operatorname{Lie}\left(G_{0}\right)$ goes through as above. Note that in most cases, $X^{p} \neq 0$.

\section{Commutative subalgebras}

In this section we study the case where $p$ is a torsion prime for an irreducible component of the root system of $G$. In each case we construct a 3-dimensional commutative restricted subalgebra of $\mathfrak{g}$ spanned by nilpotent elements $e$, $X, Y$, with $e^{p}=X^{p}=Y^{p}=0$, which lies in no Borel subalgebra of $G$. It suffices to consider the case where $G$ is simple. In what follows we will use the Bala-Carter-Pommerening notation for nilpotent orbits in $\mathfrak{g}$.

The case $p=2$.

Here we take $e$ to be an element of type $A_{1}^{3}$ if $G$ is of type $D_{\ell}$ or $E_{\ell}$, of type $A_{1} \times \tilde{A}_{1}$ if $G$ is of type $B_{\ell}$ or $F_{4}$, and of type $\tilde{A_{1}}$ if $G$ is of type $G_{2}$.

If the Dynkin diagram of $G$ is simply-laced then it has a (unique) subdiagram of type $D_{4}$. We will work within this subsystem subalgebra. Set

$$
e=e_{10_{0}^{0}}+e_{00}^{1}+e_{00}^{0}, \quad X=e_{11_{0}^{0}}+e_{01}^{1}{ }_{0}^{1}+e_{01}^{0}{ }_{1}^{0}, \quad Y=f_{11_{0}^{1}}+f_{11_{1}^{0}}+f_{01}{ }_{1}^{1} .
$$


If $G$ is of type $B_{\ell}$ or $F_{4}$ then the Dynkin diagram of $G$ has a (unique) subdiagram of type $B_{3}$, which we label with roots $\beta_{1}, \beta_{2}, \beta_{3}$, where $\beta_{3}$ is short. Here we let $e=e_{\beta_{1}}+e_{\beta_{3}}, X=e_{110}+e_{011}, Y=f_{111}+f_{012}$.

Finally, if $G$ is of type $G_{2}$ then let $e=e_{\alpha_{1}}, X=e_{11}, Y=f_{21}$.

The case $p=3$.

Here either $G$ is of type $E_{\ell}, \ell=6,7,8$ or $G$ is of type $F_{4}$. We take $e$ to be an element of type $A_{2}^{2} \times A_{1}$ if $G$ is of type $E_{\ell}$ and of type $A_{1} \times \tilde{A}_{2}$ if $G$ is of type $F_{4}$. If $G$ is of type $E_{6}, E_{7}$ or $E_{8}$ then we can restrict to the (standard) subsystem of type $E_{6}$ : let

$$
\begin{aligned}
& e=e_{0} 10000+e_{01000}+e_{0}^{00010}+e_{0}^{00001}+e_{00000}, \\
& X=e_{0}^{11100}+e_{00110}+e_{00111}-e_{01100}+e_{01110}, \\
& Y=f_{11110}+f_{00111}+f_{11100}-f_{01111}+f_{01110} .
\end{aligned}
$$

If $G$ is of type $F_{4}$ then let $e=e_{\alpha_{1}}+e_{\alpha_{3}}+e_{\alpha_{4}}, X=e_{0111}+e_{1110}-e_{0120}$ and $Y=2 f_{1111}-2 f_{1120}+f_{0121}$.

The case $p=5$.

Here $G$ is of type $E_{8}$. We choose $e$ to be an element of type $A_{4} \times A_{3}$. Let

$$
\begin{aligned}
& e=e_{\alpha_{1}}+e_{\alpha_{2}}+e_{\alpha_{3}}+e_{\alpha_{4}}+e_{\alpha_{6}}+e_{\alpha_{7}}+e_{\alpha_{8}} \\
& X=e_{1111000}+2 e_{001110}+2 e_{1111100}+2 e_{0011111}+2 e_{011110}-e_{0121000}-e_{0111100} \\
& Y=f_{0} 1111110+f_{1121000}+f_{1111100}+2 f_{0011111}+\underset{1}{2 f_{0111110}}+f_{0121100}-2 f_{0111111} .
\end{aligned}
$$

Note that in each of the above cases, there exists $e_{\alpha}$ (resp. $e_{\beta}, f_{\gamma}$ ) in the expression for $e$ (resp. $X, Y$ ) such that $\alpha+\beta-\gamma=0$.

Proposition 4.1. Let $\mathfrak{h}=k e+k X+k Y$, with $e, X, Y$ as above. Then $\mathfrak{h}$ is not contained in any Borel subalgebra of $\mathfrak{g}$.

Proof. Suppose $\mathfrak{h}$ is contained in a Borel subalgebra. Then for some $g \in G$, $\operatorname{Ad} g(\mathfrak{h}) \subset \mathfrak{b}$, where $\mathfrak{b}$ is the Borel subalgebra corresponding to the positive Weyl chamber. By the Bruhat decomposition, we have $g=u^{\prime} n u$, where $u, u^{\prime} \in U^{+}$and $n \in N_{G}(T)$. But now $\operatorname{Ad} g(\mathfrak{h}) \subset \mathfrak{b}$ if and only if $\operatorname{Ad}(n u)(\mathfrak{h}) \subset \mathfrak{b}$, thus we may assume that $u^{\prime}=1$. Let $w=n T \in W$. We will explain our argument for the case where $G$ is of type $D_{4}$ and $p=2$. Note that $\operatorname{Ad} u(e)=e+x$, where $x$ is in the span of all positive root subspaces for roots of length greater than 1 . Thus $\operatorname{Ad} n u(e) \in \mathfrak{b}$ implies, in particular, that $w\left(\alpha_{1}\right) \in \Phi^{+}$. Applying a similar argument to $X$ and $Y$, we see that $w\left(\alpha_{2}+\alpha_{3}\right) \in \Phi^{+}$and $w\left(-\left(\alpha_{1}+\right.\right.$ $\left.\left.\alpha_{2}+\alpha_{3}\right)\right) \in \Phi^{+}$. Taking the sum $w\left(\alpha_{1}\right)+w\left(\alpha_{2}+\alpha_{3}\right)+w\left(-\left(\alpha_{1}+\alpha_{2}+\alpha_{3}\right)\right)=0$, we have a contradiction. This argument works for all the examples given above, using the observation that if $e_{\alpha}$ and $e_{\beta}$ have non-zero coefficients in the expression for $e$ then $\alpha$ and $\beta$ are not congruent modulo the subgroup $\mathbb{Z} \Phi$ (and similarly for $X, Y$ ).

Finally, the examples of Section 3 and Proposition 4.1 give the following result:

Theorem 4.2. Let $G$ be a semisimple algebraic group over $k$ and $p$ a torsion prime for $G$. Then there exists a nonreduced unipotent subgroup scheme of $G$ which does not lie in any Borel subgroup of $G$.

We conclude with one further proposition which describes to some extent the nature of the 3-dimensional subalgebras defined above.

Proposition 4.3. Let $e, X$ and $Y$ be as in Proposition 4.1. Any non-zero element of $\mathfrak{h}=k e \oplus k X \oplus k Y$ is conjugate to $e$ and $N_{G}(\mathfrak{h}) / C_{G}(\mathfrak{h}) \cong \mathrm{SL}(3, k)$.

Proof. In each case, $e$ is a regular nilpotent element in $\operatorname{Lie}((L, L))$, for some Levi factor $L$ of $G$ normalized by $T$. Note that $(L, L)$ is a commuting product of type $A_{m}$ subgroups and hence $p$ is good for $(L, L)$. We choose $\tau$ to be a cocharacter of $(L, L)$ (and hence a cocharacter of $G$ ), associated to $e$ (see [5, 5.3]). In particular $e \in \mathfrak{g}(2 ; \tau)$. Then one 
checks that $\mathfrak{g}(\tau ;-1) \cap C_{\mathfrak{g}}(e)=k X \oplus k Y$. This then implies that the group $C=C_{G}(e) \cap C_{G}\left(\tau\left(k^{\times}\right)\right)$normalizes $\mathfrak{h}$. It can be checked that the adjoint representation induces a surjective morphism $C \rightarrow \operatorname{SL}(k X \oplus k Y)$. But we can apply a similar argument to an analogous subgroup of $C_{G}(Y)$. Thus $N_{G}(\mathfrak{h})$ contains the subgroups $\operatorname{SL}(k e \oplus k X)$ and $\operatorname{SL}(k X \oplus k Y)$, and hence contains $\operatorname{SL}(\mathfrak{h})$. In particular, all non-zero elements of $\mathfrak{h}$ are conjugate by an element of $N_{G}(\mathfrak{h})$. It follows from our remark on root elements in the expressions for $e, X$ and $Y$ that there can be no cocharacter in $G$ for which $e, X$ and $Y$ are all in the sum of positive weight spaces. This then implies that $N_{G}(\mathfrak{h}) / C_{G}(\mathfrak{h})$ is isomorphic to $\operatorname{SL}(\mathfrak{h})$.

\section{Acknowledgements}

We wish to thank Alexander Premet for communicating a proof of Theorem 2.2 in the case of very good primes and Jean-Pierre Serre for several useful suggestions.

\section{References}

[1] A. Borel, Linear Algebraic Groups, second edition, Graduate Texts in Mathematics, vol. 126, Springer, 1991.

[2] N. Bourbaki, Groupes et algèbres de Lie, IV, V, VI, Hermann, Paris, 1968.

[3] J. Draisma, H. Kraft, J. Kuttler, Nilpotent subspaces of maximal dimension in semisimple Lie algebras, Compos. Math. 142 (2006) $464-476$.

[4] J.E. Humphreys, Linear Algebraic Groups, Graduate Texts in Mathematics, vol. 21, second edition, Springer, 1981.

[5] J.C. Jantzen, Nilpotent orbits in representation theory, Part I, in: Lie Theory: Lie Algebras and Representations, in: Progress in Mathematics, vol. 228, Birkhäuser, 2004.

[6] M.W. Liebeck, G.M. Seitz, The maximal subgroups of positive dimension in exceptional algebraic groups, Mem. Amer. Math. Soc. 802 (2004) $1-227$.

[7] A. Premet, Nilpotent orbits in good characteristic and the Kempf-Rousseau theory, J. Algebra 260 (2003) 338-366.

[8] M. Raynaud, Groupes algébriques unipotents. Extensions entre groupes unipotents et groupes de type multiplicatif, SGA 3, Schémas en Groupes II, exposé XVII, LN 152, Springer-Verlag, 1970, pp. 532-631.

[9] K. Shinoda, The conjugacy classes of Chevalley groups of type $\left(F_{4}\right)$ over finite fields of characteristic 2, J. Fac. Sci. Univ. Tokyo Sect. IA Math. 21 (1974) 133-159.

[10] T.A. Springer, R. Steinberg, Conjugacy classes, in: Seminar on Algebraic Groups and Related Finite Groups, in: SLN, vol. 131, SpringerVerlag, 1970, pp. 168-266.

[11] R. Steinberg, Torsion in reductive groups, Adv. Math. 15 (1975) 63-92.

[12] A. Vasiu, Normal, unipotent subgroup schemes in reductive groups, C. R. Acad. Sci. Paris, Ser. I 341 (2005) $79-84$. 